Research Article

\title{
Biomechanical Effects of Aspect Ratio of the Knee during Outside-In Anterior Cruciate Ligament Reconstruction Surgery
}

\author{
Tae Soo Bae $\mathbb{D},{ }^{1}$ Byeong Chan Cho, ${ }^{1}$ and Dai-Soon Kwak $\mathbb{D}^{2}$ \\ ${ }^{1}$ Department of Biomedical Engineering, Jungwon University, 85, Munmu-ro, Goesan-eup, Goesan-gun, \\ Chungcheongbuk-do 28024, Republic of Korea \\ ${ }^{2}$ Catholic Institute for Applied Anatomy, Department of Anatomy, College of Medicine, The Catholic University of Korea, 222, \\ Banpo-daero, Seocho-gu, Seoul 06591, Republic of Korea
}

Correspondence should be addressed to Dai-Soon Kwak; daisoon@catholic.ac.kr

Received 1 July 2021; Accepted 20 August 2021; Published 6 September 2021

Academic Editor: Konstantinos Anagnostakos

Copyright (c) 2021 Tae Soo Bae et al. This is an open access article distributed under the Creative Commons Attribution License, which permits unrestricted use, distribution, and reproduction in any medium, provided the original work is properly cited.

\begin{abstract}
We analyzed tunnel length, graft bending angle, and stress of the graft according to tunnel entry position and aspect ratio (ASR: ratio of anteroposterior depth to mediolateral width) of the articular surface for the distal femur during single-bundle outside-in anterior cruciate ligament reconstruction (ACLR) surgery. We performed multiflexible body dynamic analyses with four ASR (98, 105,111 , and 117\%) knee models. The various ASRs were associated with approximately $1 \mathrm{~mm}$ changes in tunnel length. The graft bending angle increased when the entry point was far from the lateral epicondyle and was larger when the ASR was smaller. The graft was at maximum stress, $117 \%$ ASR, when the tunnel entry point was near the lateral epicondyle. The maximum stress value at a $5 \mathrm{~mm}$ distance from the lateral epicondyle was 3.5 times higher than the $15 \mathrm{~mm}$ entry position, and the cases set to $111 \%$ and $105 \%$ ASR showed 1.9 times higher stress values when at a $5 \mathrm{~mm}$ distance compared with a $15 \mathrm{~mm}$ distance. In the case set at $98 \%$ ASR, the low-stress value showed a without-distance difference from the lateral epicondyle. Our results suggest that there is no relationship between the ASR and femoral tunnel length. A smaller ASR causes a higher graft bending angle, and a larger ASR causes greater stress in the graft.
\end{abstract}

\section{Introduction}

The anterior cruciate ligament (ACL) in the knee joint is a frequently damaged ligament. An injured ACL has been reported to not only cause functional instability of the knee joint but also cause damage to the articular cartilage and secondary injuries to the peripheral tissue of the knee joint $[1$, 2]. Anterior cruciate ligament reconstruction (ACLR) is often necessary to restore an injured ACL and to restore patients to their normal activities. To date, clinical studies have not shown that ACLR reliably prevents cartilage lesions and restores knee stability to normal values $[3,4]$. The femoral tunnel position should be considered as an important parameter when planning ACLR to restore knee stability $[5,6]$.

Various techniques for creating a femoral tunnel for ACLR have been proposed and used, but the outside-in technique is used widely due to its various advantages [7]. The outside-in tunnelling technique uses a specially designed indicator to determine direction and entry point. The instrument indicates the ACL footprint and the tunnel entry point at the lateral epicondyle. The surgeon creates a tunnel by drilling in the direction guided by the instrument, and the operator can adjust the tunnel entry position at the lateral epicondyle. Therefore, many studies have been conducted on the effect of tunnel position on surgical outcome, and the femoral tunnel position is an important parameter when planning ACLR to restore knee stability [8]. Also, the femoral tunnel position has been reported to influence biomechanical characteristics of an implanted graft, and it affects knee function during postoperative rehabilitation [9]. Studies have been conducted to determine the optimal femoral tunnel position during ACL surgery. Shino et al. attempted to determine the anatomical femoral tunnel position of an implanted graft using arthroscopy without bone incision in patients with chronic ACL insufficiency [10]. Zauleck et al. 
analyzed the ACL footprint site based on the shape of the lateral intercondylar ridge and the lateral bifurcate ridge [11]. Nam-Ki and Jong-Min compared ACLR surgical methods using the transtibial, anteromedial portal, and outside-in techniques to determine the optimal femoral tunnel position [12]. However, these studies had limitations. They only performed static analyses or simulations at certain positions.

To address this issue, Kang and Bae conducted a study to determine the femoral tunnel position under continuous knee movement during ACLR surgery, although only one knee model was used [6]. In contrast, there are numerous studies of knee morphology in anatomy and orthopaedics $[13,14]$. Many morphological studies have been conducted to design an optimal artificial joint shape. Sex differences, population differences, and size mismatch were mainly studied, and the studies addressed the issue of anatomical characteristics, aspect ratio (ASR: ratio of anteroposterior depth to mediolateral width, as shown in Figure 1), and differences among the knee $[15,16]$. However, most of the biomechanical studies were conducted without considering the knee ASR. The objective of this study was to analyze the biomechanical effect of an implanted ACL graft by determining the tunnel position according to the ASR of the distal femur during flexion-extension motion. It was hypothesized that the ASR is a major factor affecting the biomechanical stability of ACLR surgery.

\section{Materials and Methods}

This study was conducted in compliance with the law about the Act on Dissection and Preservation of Corpses of the Republic of Korea (act number: 14885). All methods were performed in accordance with the relevant guidelines and regulations from the Catholic Institute for Applied Anatomy (Project identification number: R19-A027). The CT images used in this study were selected from the Catholic Digital Human Library, which was constructed by CT scans of the cadaver with the approval of the Institutional Review Board of College of Medicine, the Catholic University of Korea (No.: CUMC10U161). Written informed consent for the use of the cadaver and consent for the use of future research on the related materials were provided by all donors or authorized representatives.

2.1. Materials and Aspect Ratio Analysis. To analyze biomechanical characteristics according to the ASR of the knee joint, samples were selected from the Catholic Digital Human Library. The Catholic Digital Human Library is a collection of CT images from whole bodies of cadavers [17-19]. Only male samples were selected to exclude the effects of gender $[15,20]$, and 89 samples were selected for measurement. The mean age was $50.73 \pm 9.99$ years, and the mean height was $165.22 \pm 6.30 \mathrm{~cm}$. To generate the CT images, the slice thickness was set to $0.75-1.0 \mathrm{~mm}$ and the pixel value was set to $0.345-0.832 \mathrm{~mm}$. The obtained images were reconstructed into $3 \mathrm{D}$ skeletal models using an image based on a 3D reconstruction program (Mimics, Ver. 20; Materialize, Belgium). Samples showed no congenital anom-

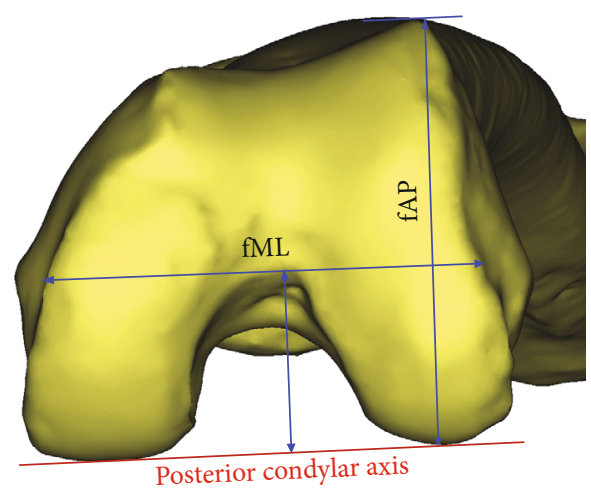

FIgURE 1: Measurement parameters for calculating the ASR. fAP indicates the height of the lateral condyle, and $\mathrm{fML}$ is the mediolateral width at the intercondylar notch parallel to the posterior condylar axis. ASR is defined as $\mathrm{fML} / \mathrm{fAP} \times 100$.

alies or pathological deformities around the knee joint. The maximum length parallel to the posterior condylar line was defined as the mediolateral (fML) width, whereas the maximum lengths of the lateral femoral condyles perpendicular to the posterior condylar line were defined as lateral anteroposterior (fAP) heights, respectively (Figure 1). The ASR at the distal femur was defined as the ratio of the height of the lateral condyles and the maximal mediolateral width $(\mathrm{fML} / \mathrm{fAP} \times 100$, Figure 1$)$. A small ASR indicates a circular knee shape, and a large ASR indicates a mediolateral long elliptical shape. After assessing the ASR range of the target models, we categorized the models into four stages through histogram analysis. The four representative models were selected for each group and used as analytical models.

\subsection{Kinematical Tracking for Continuous Flexion/Extension} of the Knee Joint. By capturing continuous movements of knee flexion/extension and inputting the trajectory data for knee movements to a computational analysis model, we aimed to implement a continuous knee movement that is different from the discrete knee movements reported in the existing studies. First, the shape of the cadaver femur and tibia was reconstructed with a 3D laser scanner (Faro Arm, Lake Mary, FL, USA) [6]. Four male cadaver knees were used. The mean age of the cadavers was 52.25 (44 60) years, and the mean height was $167.25(163 \sim 171) \mathrm{cm}$. To accurately track knee flexion and extension motions, three markers were attached to major landmarks on each femur and tibia: the lateral collateral ligament attachment point, the medial collateral ligament attachment point, and the popliteal muscle-tendon complex. The kinematical data for each marker were captured at $15^{\circ}$ intervals using motion capturing and image processing software (Geomagic 10, 3D System, Morrisville, USA). The 3-dimensional positions of each marker were fit to the shape of the femur and knee. Continuous knee movements were then extracted from discrete kinematical data measured at 15-degree intervals using the 5th-order spline interpolation technique to avoid undesired oscillation of the kinematic data during the dynamic simulation. During knee flexion and extension, the gap 
between the articular surfaces of the tibia and the femur was set at $2 \mathrm{~mm}[21,22]$.

\subsection{Three-Dimensional Multiflexible Body Dynamic Model} and Femoral and Tibial Tunnel Placement. In this study, a reconstructed three-dimensional knee joint model based on CT images was implemented using Mimics 20.0 (Materialize, Leuven, Belgium), and a dynamic analysis model was generated that applied kinematical tracking data for the knee that was already secured in the cadaver. In addition, this study is aimed at gaining insight into the optimal surgical site by analyzing the stress applied to the implanted ACL graft considering ASR of the knee joint under continuous motion. Therefore, the dynamic analysis model implemented in this study was made into a multiflexible body dynamic (MFBD) model that reduced analysis time by setting the implanted ACL graft as a deformable flexible body (finite element model) capable of being analyzed for stress effects, and the implanted ACL graft was set as a rigid body. For this, a model capable of MFBD analysis was implemented using a commercial analysis program (RecurDyn V9R1, FunctionBay, Korea) [6].

Next, in order to apply the outside-in surgical technique to the implemented 3D MFBD model, we drilled a surgical tunnel with $8 \mathrm{~mm}$ diameter suitable for the femur and tibia, respectively, by using the analysis program's editing tool. A tibial tunnel was created through the proximal crosssection using the anatomical axis measurement method on anthropometric measurements, and the femoral footprint was obtained from the sagittal plane using Bernard et al.'s quadrant method $[23,24]$. The starting point for the three investigated femoral tunnels was the $45^{\circ}$ posterior-proximal angle with distances of $5 \mathrm{~mm}$ (A), $10 \mathrm{~mm}$ (B), and $15 \mathrm{~mm}$ (C) from the lateral femoral epicondyle, as recommended by our previous analyses (Figure 2) [6].

\subsection{Multiflexible Body Dynamic Analyses for Outside-In} ACLR Surgeries. Among ACLR techniques, the outside-in method was adopted in this study. For this, both ends of the graft were attached to the MFBD model with the femoral and tibia tunnels. First, the implanted single-bundle ACL graft was modeled as a cylindrical flexible body with a diameter of $7.9 \mathrm{~mm}$ and a length of $100 \mathrm{~mm}$, respectively [23,25]. As mentioned earlier, the implanted ACL graft was designed as a flexible body (finite element model), and the number of elements was 5,594, the number of nodes was 6,520 , and the element size was $1 \mathrm{~mm}$. The material properties of the implanted ACL graft were set to $111 \mathrm{MPa}$ Young's modulus and 0.46 Poisson ratio referred to existing studies $[6,26]$. Next, the surface contact condition between the graft and the inside of each tunnel was applied to avoid penetration of each other. Since the implanted ACL graft was modeled as a flexible body and the tunnels of the femur and tibia as rigid bodies, the contact conditions were based on Hertz contact theory to avoid boundary overlap. The contact stiffness and contact damping were set to $10 \mathrm{~N} / \mathrm{mm}$ and 0.0001 , respectively, at which the boundaries between the flexible and rigid bodies did not overlap. The flexible body model was fixed at the beginning of the tibial tunnel and femoral tunnel using spring and bushing elements, respectively. A bushing force (stiffness $226 \mathrm{~N} / \mathrm{mm}$ ) was applied functionally to replace an interference screw at the starting point of the tibial tunnel [27]. To prepare for a case in which the graft was short, a button fixture (stiffness $1589.9 \mathrm{~N} / \mathrm{mm}$ ) with a $20 \mathrm{~mm}$ loop was connected to the implanted graft at the femoral tunnel starting point $[6,28]$. This rigid body knee joint model with deformable ACL was based on our previous analyses and was validated through those studies [6].

For dynamic simulation, MFBD analyses by using simulation package (Recurdyn V9R1, FunctionBay, Korea) for 12 cases were performed by simulating four different ASR knee models $(84 \%, 88 \%, 93 \%$, and $97 \%)$ at three recommended tunnel entry points (posterior-proximal sites at 5, 10, and $15 \mathrm{~mm}$ distances from the lateral femoral epicondyle; Figure 2). Then, we compared the calculated von Mises stresses of the ACL graft, the femoral tunnel lengths, and the graft bending angles for each placement of the implanted graft in the knee models (Figure 3).

\section{Results}

3.1. Aspect Ratio of the Distal Femur. The average mediolateral (fML) width was $69.32 \pm 3.50 \mathrm{~mm}$. The average lateral anteroposterior (fAP) height was $64.63 \pm 3.65 \mathrm{~mm}$. The calculated ASR was from $95 \%$ to $119 \%$ (Figure 4). We selected ASRs at $98 \%, 105 \%, 111 \%$, and $117 \%$ for biomechanical analysis because they represented quartile values across the distribution of knee samples.

3.2. Length of Femoral Tunnel. Regarding femoral tunnel lengths of the four different ASR knee models, the shortest femoral tunnel was $27.22 \mathrm{~mm}$ at $45^{\circ}$ of the posteriorproximal direction at a distance of $15 \mathrm{~mm}(\mathrm{C})$ and an ASR of $111 \%$, whereas the longest femoral tunnel was $34.25 \mathrm{~mm}$ at a distance of $5 \mathrm{~mm}(\mathrm{~A})$ and an ASR of $105 \%$. The average femoral tunnel lengths were $32.93 \mathrm{~mm}, 30.34 \mathrm{~mm}$, and $28.11 \mathrm{~mm}$ when the distance was $5 \mathrm{~mm}$ (A), $10 \mathrm{~mm}$ (B), and $15 \mathrm{~mm}(\mathrm{C})$, respectively. Femoral tunnel length decreased as the distance increased at the $45^{\circ}$ posteriorproximal direction from the lateral femoral epicondyle. The average femoral tunnel lengths were $29.99 \mathrm{~mm}$, $31.74 \mathrm{~mm}, 29.50 \mathrm{~mm}$, and $30.60 \mathrm{~mm}$ at ASRs of $98 \%, 105 \%$, $111 \%$, and $117 \%$, respectively (Figure 5).

3.3. Bending Angle of the Implanted Graft. Regarding the graft bending angles of the four different ASR knee models, the smallest graft bending angle was $100.85^{\circ}$ with a distance of $5 \mathrm{~mm}$ (A) and an ASR of $111 \%$, whereas the largest graft bending angle was $123.53^{\circ}$ with a distance of $15 \mathrm{~mm}$ (C) and an ASR of $98 \%$. The average graft bending angles were $103.52^{\circ}, 111.55^{\circ}$, and $120.4^{\circ}$ when the tunnel entry positions were $5 \mathrm{~mm}(\mathrm{~A}), 10 \mathrm{~mm}(\mathrm{~B})$, and $15 \mathrm{~mm}(\mathrm{C})$, respectively. The graft bending angle increased as the distance increased from the lateral femoral epicondyle. The average graft bending angles at ASRs of $98 \%, 105 \%, 111 \%$, and $117 \%$ were $114.75^{\circ}, 112.40^{\circ}, 109.64^{\circ}$, and $110.51^{\circ}$, respectively (Figure 6).

3.4. von Mises Stress of Implanted Grafts during Continuous Knee Motion. Regarding the von Mises stress of the implanted 

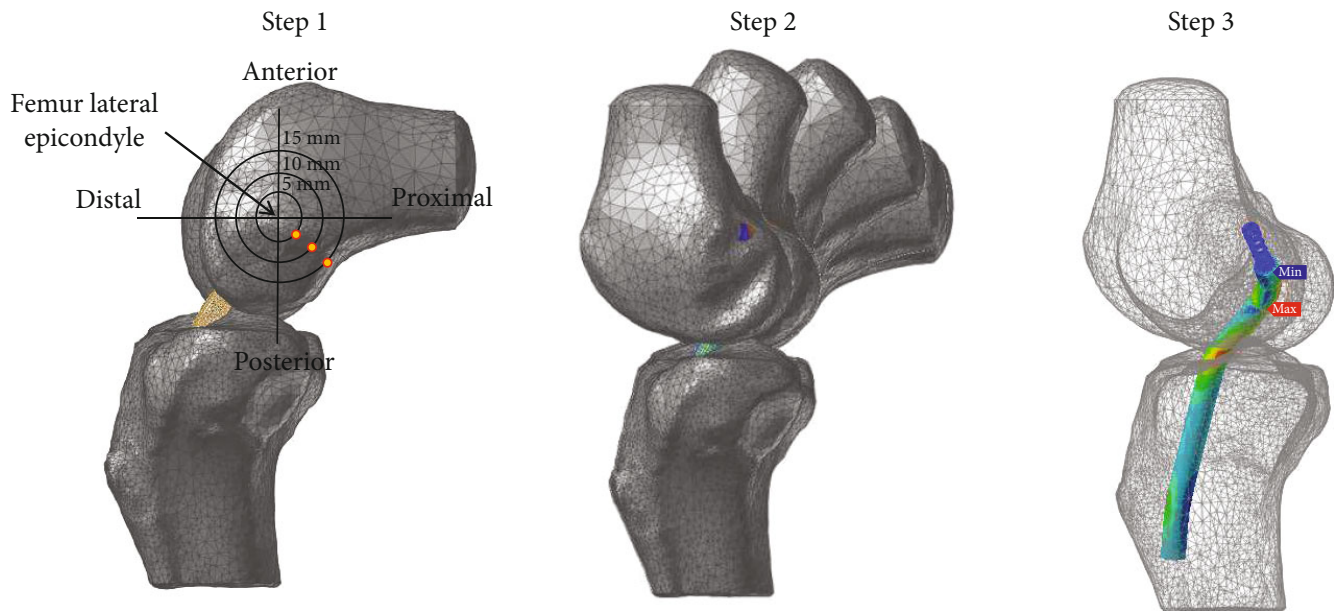

Figure 2: The process of computational analysis on ACLR for the 3D knee model showing recommended tunnel entry points during continuous motions; Step 1: the starting point for the three investigated femoral tunnels was the $45^{\circ}$ posterior-proximal angle at $5 \mathrm{~mm}$ (A), $10 \mathrm{~mm}$ (B), and $15 \mathrm{~mm}$ (C) distances from the lateral epicondyle. Step 2: follow the shape of the knee using motion analysis data. Step 3: calculate stresses of the implant.

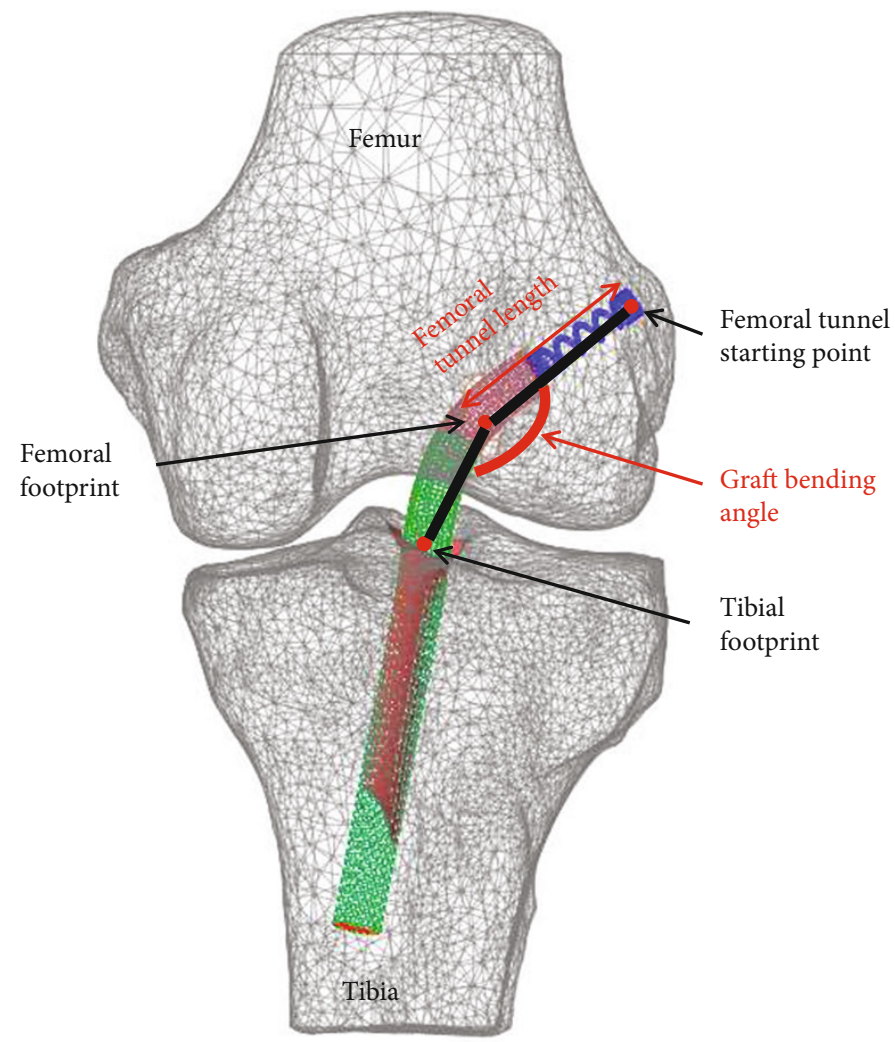

FIGURE 3: Description of the graft bending angle and femoral tunnel length.

grafts in the four different ASR knee models during continuous knee movement, the lowest von Mises stress was 19.4 $\mathrm{MPa}$ at the $45^{\circ}$ posterior-proximal angle at a distance of $15 \mathrm{~mm}(\mathrm{C})$ and an ASR of 98\%, whereas the highest von Mises stress was $71 \mathrm{MPa}$ at the $45^{\circ}$ posterior-proximal angle with a distance of $5 \mathrm{~mm}$ (A) and an ASR of $117 \%$ (Figure 7). The von Mises stress of the implanted graft decreased with increas- ing distance at ASRs of $105 \%, 111 \%$, and $117 \%$, but the von Mises stress of the implanted graft at an ASR of $98 \%$ changed very little as the distance from the lateral epicondyle increased. The von Mises stress of the implanted graft during continuous knee movement also increased as the knee joint angle decreased. The highest stress value was observed when the knee joint angle was between $0^{\circ}$ and $30^{\circ}$ (Figure 8 ). 


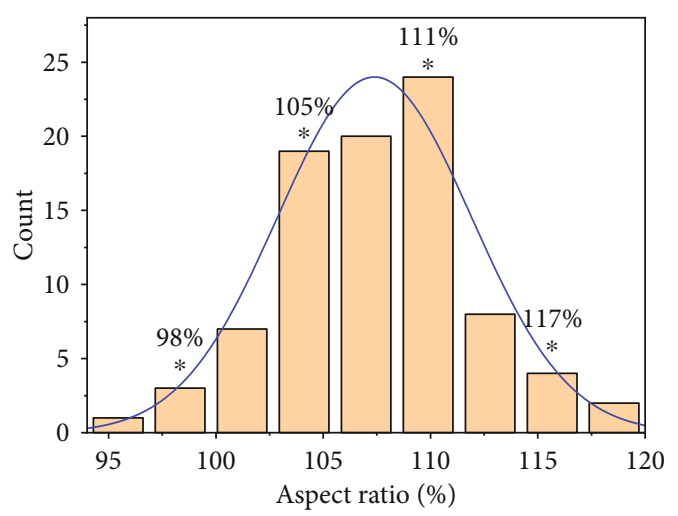

Figure 4: Histogram of knee ASRs (total 89 samples); simulation samples were selected at $98 \%, 105 \%, 111 \%$, and $117 \%$.

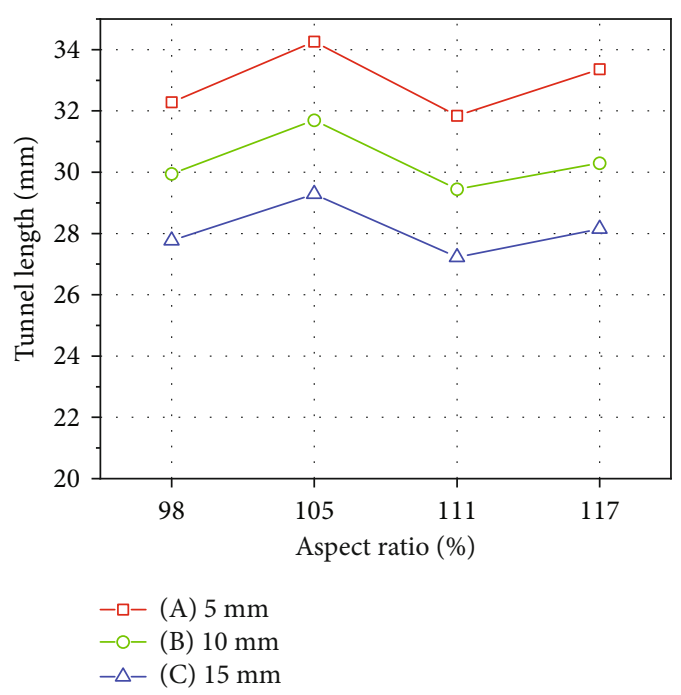

FIGURE 5: Calculated femoral tunnel length of patient-derived ACL graft at $5 \mathrm{~mm}, 10 \mathrm{~mm}$, and $15 \mathrm{~mm}$ tunnel entry point distance from the lateral epicondyle. Femoral tunnel length decreased as the tunnel entry point distance from the lateral femoral epicondyle increased.

\section{Discussion}

The main cause of ACLR surgery failure is incorrect tunnel position $[29,30]$. This mispositioning causes damage to knee structures (cartilage, ligaments, meniscus, etc.) and could lead to arthritis [31]. The main purpose of ACL surgery is stabilization to prevent early knee wear. If the femoral tunnel is placed anteriorly, it causes tension in the graft, limiting flexion and rotational stability [32]. To reduce failure and improve stability during ACLR surgery, surgical methods considering anatomy, such as the outside-in technique, have been introduced $[7,33]$. In this study, tunnel position was selected based on the ASR of the knee through the outsidein technique during ACL surgery.

For successful ACL reconstruction, many researchers have focused on the optimal tunnel position with tunnelling technique through biomechanical simulations $[6,25,34,35]$. However, anatomical characteristics of the knee were not

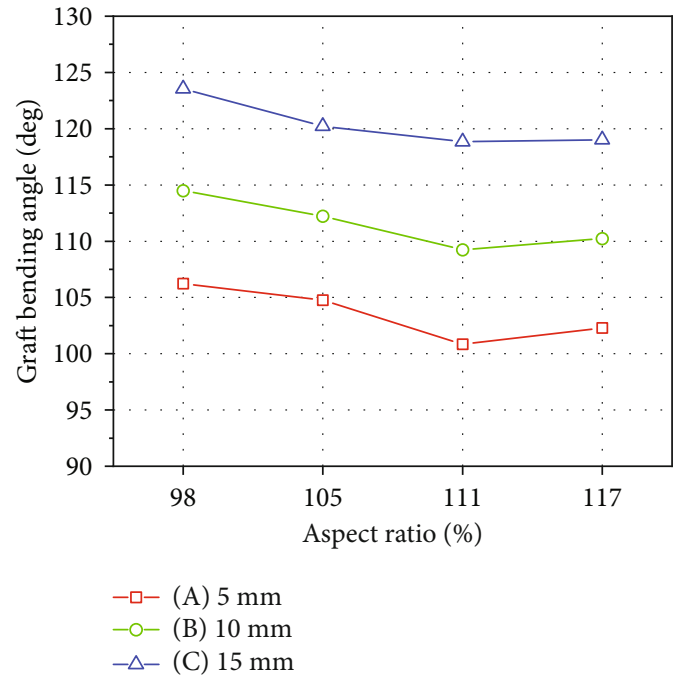

FIgURE 6: Calculated graft angles of ACL reconstruction at $5 \mathrm{~mm}$, $10 \mathrm{~mm}$, and $15 \mathrm{~mm}$ tunnel entry point distances from the lateral epicondyle. The graft bending angle increased as the tunnel entry point distance from the lateral femoral epicondyle increased.

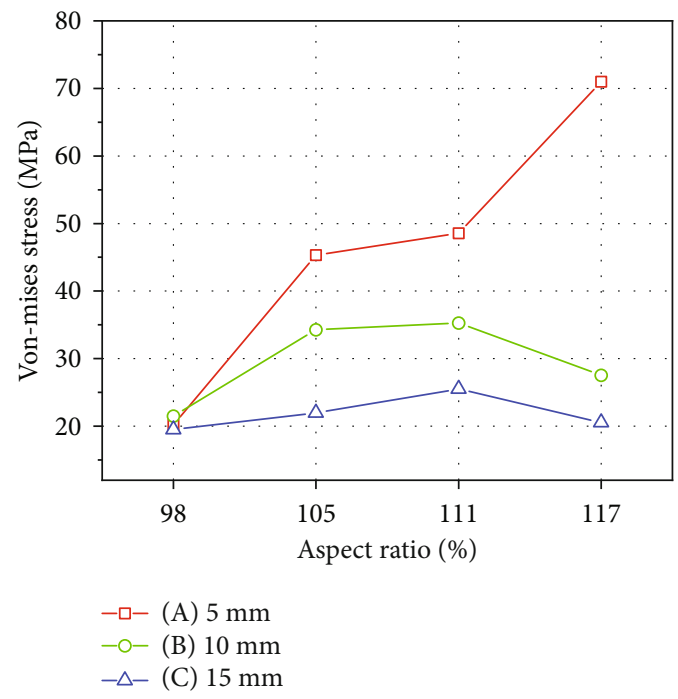

Figure 7: Maximum von Mises stress in grafts according to knee ASR. Stress decreased with increasing distance at ASRs of $105 \%$, $111 \%$, and $117 \%$. The stress at an ASR of $98 \%$ changed very little with increasing distance from the lateral epicondyle.

considered in these studies. Computational studies of the optimal tunnel position mentioned above have been carried out without any consideration of these anatomical characteristics. Therefore, in this study, we investigated the biomechanical effect of the implanted graft on the optimal tunnel position for four representative ASRs.

According to Beckers et al. [36], men had wider knees (range 108 151\%) than women (range 106 146\%), and compared to Caucasian knees (range 116 126\%), Arabian (range 106 149\%) and Indian (range 118 147) knees were wider, while East Asian knees were narrower (range 100 118). This study on Korean men showed knee ASRs 


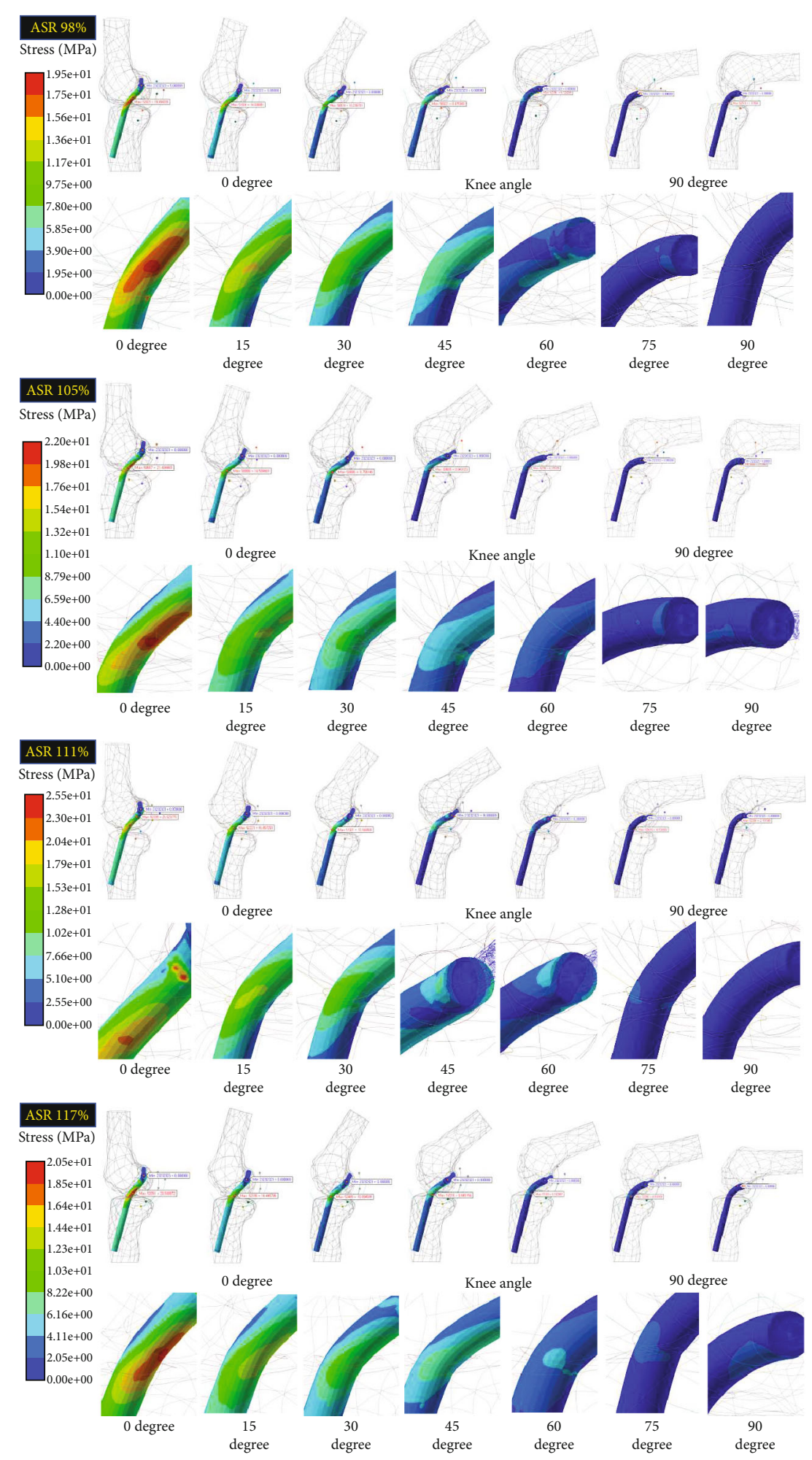

Figure 8: Calculated von Mises stress contour of implanted ACL grafts at seven major angles during continuous knee flexion/extension movement as a result of MFBD analyses at the various ASRs: Max. stress (red) and Min. stress (blue).

of $95 \sim 119 \%$, similar to the results of a previous study on Asians [37, 38]. The femoral tunnel length for ACL graft was not affected by the ASR of the distal femur. The femoral tunnel length at $111 \%$ ASR showed a minor decrement compared with $117 \%$ ASR. The tunnel length at 105\% ASR increased and then decreased at 98\% ASR. Finally, the tunnel length at $117 \%$ ASR was similar to the length at $98 \%$ ASR (Figure 6). The changes in tunnel length among the various ASRs were about $1 \mathrm{~mm}$, and the shortest tunnel length was $27.22 \mathrm{~mm}$. Existing studies found that the 
minimum required tunnel length was $25 \mathrm{~mm}$ for successful reconstruction surgery $[35,39]$. Thus, the ASR of the distal femur might not be an essential factor for determining adequate tunnel length.

The calculated value of the graft bending angle showed an increase when the entry point was far from the lateral epicondyle. The results at $111 \%$ and $117 \%$ ASRs were similar or were slightly decreased compared with the increase at $98 \%$ and $105 \%$ ASRs (Figure 7). The graft bending angle had a larger value as ASR decreased at every entry point. Thus, surgeons should pay attention to the graft bending angle when confronted with a knee with a small ASR. A small ASR causes a large value for the graft bending angle, and the graft bending angle affects early graft healing and tunnel widening [40-42].

Based on the review of previous computational studies, accurate comparisons were limited due to the lack of information on the ASRs of the analysis models. According to the study of finite element analysis by Song et al. [43], the von Mises stress distribution of a single bundle was 16.5 to $24 \mathrm{MPa}$. In addition, Westermann et al. performed FEA studies about the various diameters (5 to $9 \mathrm{~mm}$ ) of ACL grafts with a knee angle of 20 degrees [44]. Their results showed a von Mises stress distribution of 15 to $22 \mathrm{MPa}$. Lastly, in the study of Wan et al. [45], a three-dimensional FEA analysis of ACL reconstruction was performed using three grafts at a knee angle of 0 degrees. As a result, the stress distribution was 20 to $52 \mathrm{MPa}$. Our study showed the highest stress of the implanted ACL graft at a knee angle of 0 to 15 degrees for the four ASR models. In addition, our study showed calculated stress values (19.49 to $71 \mathrm{MPa})$ in the range of those from previous studies because various tunnel positions and ASRs were considered in the analysis.

The maximum stress in the graft, when the ASR was $117 \%$, had a high-stress value with the tunnel entry point near the lateral epicondyle. The maximum stress value at a $5 \mathrm{~mm}$ distance from the lateral epicondyle was 3.5 times higher than the $15 \mathrm{~mm}$ entry position, and in the cases at $105 \%$ and $111 \%$ ASR, the stress was 1.9 times higher at a $5 \mathrm{~mm}$ distance compared with a $15 \mathrm{~mm}$ distance. Otherwise, in cases at $98 \%$ ASR, a low-stress value was achieved at all entry points. When reconstructing the ACL with a large ASR, creating a femoral tunnel entry point close to the lateral epicondyle can increase stress on the graft.

The differences in results were small in the ASR 105$111 \%$ range, which accounted for most of the sample. However, the difference of the bending angle was observed with a large ASR of $117 \%$, which is close to an ellipse, and the maximum stress increased at small ASR (98\%), which is close to a circular shape. Considering the number of samples in this study (89 total), an $117 \%$ ASR was found in $10.11 \%(9 / 89)$ of the total sample, and a $98 \%$ ASR was found in $8.89 \%(8 / 89)$. Therefore, about $20 \%$ of the samples had a small or large ASR; at least in these samples, the difference in ASR should be considered during ACLR surgery.

This study had some limitations. First, we had to use cadavers to analyze knee kinematics in the flexionextension process. Passive movement was performed without consideration of body weight. In addition, major liga- ments and soft tissues such as the medial/lateral collateral ligament, posterior cruciate ligament, and anterolateral ligament were not considered. However, the main role of these soft tissues is involvement in passive movement. Second, the results were not validated by biomechanical testing after computational analysis of graft tensioning according to ASR and tunnel entry position. Such analysis was not possible here. A biomechanical test with three recommended tunnel entry points with one ASR requires at least three cadavers with the same ASR. In addition, it is difficult to produce three tunnels in a femur with the same ASR. However, this study confirmed that kinematic data obtained from limited conditions and various ASRs of the knee can affect movement, and these factors can affect ACL graft in small or large ASRs.

\section{Conclusions}

This study is aimed at confirming through computational analysis whether ASR, which was not examined in previous studies, should be considered for current surgical procedures. Our computations showed that there is no relationship between ASR and femoral tunnel length for ACLR surgery. A smaller ASR causes a higher graft bending angle, and a larger ASR causes greater stress to the graft. As a result, the ASR of the distal femur is a factor that should be considered in ACLR surgical plans. Small or large ASRs should be considered during ACLR surgery. We also believe that clinical studies on possible graft stress outcomes will be necessary.

\section{Data Availability}

The datasets used and/or analyzed during the current study are available from the corresponding author on reasonable request.

\section{Conflicts of Interest}

The authors declare that there is no conflict of interest regarding the publication of this paper.

\section{Acknowledgments}

This research was supported by the Basic Science Research Program through the National Research Foundation of Korea (NRF) funded by the Ministry of Education (NRF2017R1D1A3B04033410).

\section{References}

[1] T. Lien-Iversen, D. B. Morgan, C. Jensen, M. A. Risberg, L. Engebretsen, and B. Viberg, "Does surgery reduce knee osteoarthritis, meniscal injury and subsequent complications compared with non-surgery after ACL rupture with at least 10 years follow-up? A systematic review and meta-analysis," British Journal of Sports Medicine, vol. 54, no. 10, pp. 592-598, 2020.

[2] J. Kvist, S. Filbay, C. Andersson, C. L. Ardern, and H. Gauffin, "Radiographic and symptomatic knee osteoarthritis 32 to 37 
years after acute anterior cruciate ligament rupture," The American Journal of Sports Medicine, vol. 48, no. 10, pp. 2387-2394, 2020.

[3] C. L. Ardern, K. E. Webster, N. F. Taylor, and J. A. Feller, "Hamstring strength recovery after hamstring tendon harvest for anterior cruciate ligament reconstruction: a comparison between graft types," Arthroscopy, vol. 26, no. 4, pp. 462-469, 2010.

[4] C. L. Ardern, K. E. Webster, N. F. Taylor, and J. A. Feller, "Return to the preinjury level of competitive sport after anterior cruciate ligament reconstruction surgery: two-thirds of patients have not returned by 12 months after surgery," The American Journal of Sports Medicine, vol. 39, no. 3, pp. 538543,2011

[5] V. Jaecker, T. Zapf, J. H. Naendrup et al., "High non-anatomic tunnel position rates in ACL reconstruction failure using both transtibial and anteromedial tunnel drilling techniques," Archives of Orthopaedic and Trauma Surgery, vol. 137, no. 9, pp. 1293-1299, 2017.

[6] K. Kang and T. S. Bae, "Effect of femoral tunnel positions on graft stress in outside-in ACL reconstruction surgery during continuous knee motion: a simulation study," The International Journal of Medical Robotics and Computer Assisted Surgery, vol. 13, no. 4, article e1817, 2017.

[7] G. Ji, A. Han, X. Hao, N. Li, R. Xu, and F. Wang, "Better rotational control but similar outcomes with the outside-in versus the transtibial drilling technique for anterior cruciate ligament reconstruction: a systematic review of comparative trials," Archives of Orthopaedic and Trauma Surgery, vol. 138, no. 11, pp. 1575-1581, 2018.

[8] T. Iriuchishima and B. Goto, "Systematic review of surgical technique and tunnel target points and placement in anatomical single-bundle ACL reconstruction," The Journal of Knee Surgery, 2020.

[9] S. R. Lee, H. W. Jang, D. W. Lee, S. W. Nam, J. K. Ha, and J. G. Kim, "Evaluation of femoral tunnel positioning using 3dimensional computed tomography and radiographs after single bundle anterior cruciate ligament reconstruction with modified transtibial technique," Clinics in Orthopedic Surgery, vol. 5, no. 3, pp. 188-194, 2013.

[10] K. Shino, T. Suzuki, T. Iwahashi et al., "The resident's ridge as an arthroscopic landmark for anatomical femoral tunnel drilling in ACL reconstruction," Knee Surgery, Sports Traumatology, Arthroscopy, vol. 18, no. 9, pp. 1164-1168, 2010.

[11] M. K. Zauleck, S. Gabriel, M. F. Fischmeister, and L. Hirtler, "Origin of the anterior cruciate ligament and the surrounding osseous landmarks of the femur," Clinical Anatomy, vol. 27, no. 7, pp. 1103-1110, 2014.

[12] K. Nam-Ki and K. Jong-Min, "The three techniques for femoral tunnel placement in anterior cruciate ligament reconstruction: transtibial, anteromedial portal, and outside-in techniques," Arthroscopy and Orthopedic Sports Medicine., vol. 2, no. 2, pp. 77-85, 2015.

[13] H. J. Cho, D. S. Kwak, and I. B. Kim, "Morphometric evaluation of Korean femurs by geometric computation: comparisons of the sex and the population," BioMed Research International, vol. 2015, Article ID 730538, 9 pages, 2015.

[14] M. Verma, S. Joshi, A. Tuli, S. Raheja, P. Jain, and P. Srivastava, "Morphometry of proximal femur in Indian population," Journal of Clinical and Diagnostic Research, vol. 11, no. 2, pp. AC01-AC04, 2017.
[15] K. Hitt, J. R. Shurman, K. Greene et al., "Anthropometric measurements of the human knee: correlation to the sizing of current knee arthroplasty systems," Journal of Bone and Joint Surgery, vol. 85-A, Supplement 4, pp. 115-122, 2003.

[16] D. S. Kwak, S. Surendran, Y. H. Pengatteeri et al., "Morphometry of the proximal tibia to design the tibial component of total knee arthroplasty for the Korean population," The Knee, vol. 14, no. 4, pp. 295-300, 2007.

[17] S. Kim, D. S. Kwak, and I. B. Kim, "Morphometric analysis and classification of the cross-sectional shape of the C2 lamina," Biomed Research International, vol. 2017, 7 pages, 2017.

[18] S. A. Park, D. S. Kwak, and H. J. Cho, “Technical variation of trans-articular sacroiliac joint (SIJ) fusion using three screws considering the effects of sacral dysplasia in patients with non-traumatic SIJ pain," Bmc Musculoskeletal Disorders, vol. 20, no. 1, 2019.

[19] S. A. Park, D. S. Kwak, and S. L. You, "Entry zone of iliac screw fixation to maintain proper entry width and screw length," European Spine Journal., vol. 24, no. 11, pp. 2573-2579, 2015.

[20] R. P. Csintalan, M. M. Schulz, J. Woo, P. J. McMahon, and T. Q. Lee, "Gender differences in patellofemoral joint biomechanics," Clinical Orthopaedics and Related Research, vol. 402, pp. 260-269, 2002.

[21] A. Faisal, S. C. Ng, S. L. Goh, and K. W. Lai, "Knee cartilage segmentation and thickness computation from ultrasound images," Medical \& Biological Engineering \& Computing, vol. 56, no. 4, pp. 657-669, 2018.

[22] R. J. Schmitz, D. Harrison, H. M. Wang, and S. J. Shultz, "Sagittalplane knee moment during gait and knee cartilage thickness," Journal of Athletic Training, vol. 52, no. 6, pp. 560-566, 2017.

[23] M. Bernard, P. Hertel, H. Hornung, and T. Cierpinski, "Femoral insertion of the ACL. Radiographic quadrant method," The American journal of knee surgery, vol. 10, no. 1, pp. 14-21, 1997.

[24] Y. W. Ko, S. J. Rhee, I. W. Kim, and J. D. Yoo, "The correlation of tunnel position, orientation and tunnel enlargement in outside-in single-bundle anterior cruciate ligament reconstruction," Knee Surgery \& Related Research, vol. 27, no. 4, pp. 247-254, 2015.

[25] T. S. Bae and B. C. Cho, "Biomechanical effect of tunnel positions and pre-tension forces on implanted graft stress and strain during outside-in ACL reconstruction surgery: a simulation study," International Journal of Precision Engineering and Manufacturing., vol. 21, no. 3, pp. 519-524, 2020.

[26] F. R. Noyes and E. S. Grood, "The strength of the anterior cruciate ligament in humans and Rhesus monkeys," The Journal of Bone and Joint Surgery. American Volume, vol. 58, no. 8, pp. 1074-1082, 1976.

[27] K. H. Bloemker, T. M. Guess, L. Maletsky, and K. Dodd, "Computational knee ligament modeling using experimentally determined zero-load lengths," The Open Biomedical Engineering Journal, vol. 6, no. 1, pp. 33-41, 2012.

[28] C. Jin, S. V. Paluvadi, S. Lee, S. Yoo, E. K. Song, and J. K. Seon, "Biomechanical comparisons of current suspensory fixation devices for anterior cruciate ligament reconstruction," International Orthopaedics, vol. 42, no. 6, pp. 1291-1296, 2018.

[29] E. E. Khalfayan, P. F. Sharkey, A. H. Alexander, J. D. Bruckner, and E. B. Bynum, "The relationship between tunnel placement and clinical results after anterior cruciate ligament reconstruction," The American Journal of Sports Medicine, vol. 24, no. 3, pp. 335-341, 1996. 
[30] L. A. Pinczewski, L. J. Salmon, W. F. M. Jackson, R. B. P. von Bormann, P. G. Haslam, and S. Tashiro, "Radiological landmarks for placement of the tunnels in single-bundle reconstruction of the anterior cruciate ligament," Journal of Bone and Joint Surgery. British Volume (London), vol. 90, no. 2, pp. 172-179, 2008.

[31] G. Samitier, A. I. Marcano, E. Alentorn-Geli, R. Cugat, K. W. Farmer, and M. W. Moser, "Failure of anterior cruciate ligament reconstruction," The Archives of Bone and Joint Surgery, vol. 3, no. 4, pp. 220-240, 2015.

[32] F. Rayan, S. K. Nanjayan, C. Quah, D. Ramoutar, S. Konan, and F. S. Haddad, "Review of evolution of tunnel position in anterior cruciate ligament reconstruction," World Journal of Orthopedics, vol. 6, no. 2, pp. 252-262, 2015.

[33] B. N. Robin, S. S. Jani, S. C. Marvil, J. B. Reid, C. K. Schillhammer, and J. H. Lubowitz, "Advantages and Disadvantages of Transtibial, Anteromedial Portal, and Outside- In Femoral Tunnel Drilling in Single-Bundle Anterior Cruciate Ligament Reconstruction: A Systematic Review," Arthroscopy, vol. 31, no. 7, pp. 1412-1417, 2015.

[34] B. Forsythe, S. Kopf, A. K. Wong et al., "The location of femoral and tibial tunnels in anatomic double-bundle anterior cruciate ligament reconstruction analyzed by three-dimensional computed tomography models," The Journal of Bone and Joint Surgery. American Volume, vol. 92, no. 6, pp. 1418-1426, 2010.

[35] L. G. B. Guglielmetti, L. G. Shimba, L. C. do Santos et al., "The influence of femoral tunnel length on graft rupture after anterior cruciate ligament reconstruction," Journal of Orthopaedics and Traumatology, vol. 18, no. 3, pp. 243-250, 2017.

[36] L. Beckers, J. H. Müller, J. Daxhelet, M. Saffarini, T. Aït-SiSelmi, and M. P. Bonnin, "Sexual dimorphism and racial diversity render bone-implant mismatch inevitable after offthe-shelf total knee arthroplasty: a systematic review and meta-analysis," Knee Surgery, Sports Traumatology, Arthroscopy, 2021.

[37] M. Nishikawa, H. Owaki, S. Kaneshiro, and T. Fuji, "Preoperative morphometric differences in the distal femur are based on skeletal size in Japanese patients undergoing total knee arthroplasty," Knee Surgery, Sports Traumatology, Arthroscopy, vol. 22, no. 12, pp. 2962-2968, 2014.

[38] A. V. G. Reddy, S. R. Sankineani, R. Agrawal, and C. Thayi, "Comparative study of existing knee prosthesis with anthropometry of Indian patients and other races, a computer tomography 3D reconstruction-based study," Journal of Clinical Orthopaedics and Trauma, vol. 11, Supplement 2, pp. S228-S233, 2020.

[39] A. Bedi, B. Raphael, A. Maderazo, H. Pavlov, and R. J. Williams 3rd, "Transtibial versus anteromedial portal drilling for anterior cruciate ligament reconstruction: a cadaveric study of femoral tunnel length and obliquity," Arthroscopy, vol. 26, no. 3, pp. 342-350, 2010.

[40] L. Chen, Y. Wu, G. Lin et al., "Graft bending angle affects allograft tendon maturity early after anterior cruciate ligament reconstruction," Knee Surgery, Sports Traumatology, Arthroscopy, vol. 26, no. 10, pp. 3048-3054, 2018.

[41] Y. Tashiro, T. Gale, V. Sundaram et al., "The graft bending angle can affect early graft healing after anterior cruciate ligament reconstruction: in vivo analysis with 2 years' followup," The American Journal of Sports Medicine, vol. 45, no. 8, pp. 1829-1836, 2017.

[42] Y. Tashiro, V. Sundaram, E. Thorhauer et al., "In Vivo Analysis of Dynamic Graft Bending Angle in Anterior Cruciate Lig- ament- Reconstructed Knees During Downward Running and Level Walking: Comparison of Flexible and Rigid Drills for Transportal Technique," Arthroscopy, vol. 33, no. 7, pp. 1393-1402, 2017.

[43] Y. Song, R. E. Debski, V. Musahl, M. Thomas, and S. L. Woo, "A three-dimensional finite element model of the human anterior cruciate ligament: a computational analysis with experimental validation," Journal of Biomechanics, vol. 37, no. 3, pp. 383-390, 2004.

[44] R. W. Westermann, B. R. Wolf, and J. M. Elkins, "Effect of ACL reconstruction graft size on simulated Lachman testing: a finite element analysis," Iowa Orthopedic Journal, vol. 33, pp. 70-77, 2013.

[45] C. Wan, Z. Hao, and S. Wen, "The finite element analysis of three grafts in the anterior cruciate ligament reconstruction," in 2011 4th International Conference on Biomedical Engineering and Informatics (BMEI)., pp. 1338-1342, China, 2011. 